\title{
A Grain Boundary “TTT” - “Tribute to Thomas”!
}

\author{
Martin P. Harmer ${ }^{1}$, Christopher J. Marvel ${ }^{1}$ and Patrick R. Cantwell ${ }^{2}$ \\ 1. Department of Materials Science and Engineering, Lehigh University, Bethlehem PA 18015 USA \\ 2. Department of Mechanical Engineering, Rose-Hulman Institute of Technology, Terre Haute IN 47803 \\ USA
}

I had the good fortune to study electron microscopy at UC Berkeley during my graduate studies under the expert guidance of Gareth Thomas. He was a true master of the electron microscope and had an incredible gift for applying electron microscopy to solve critical problems. My year-long fellowship under Gareth was a pivotal experience in my life and had a profound influence on my conception of how materials science research should be conducted. I am deeply grateful to Gareth for his influence on my development as a materials scientist and I truly believe that he deserves recognition for his positive impact on an entire generation of materials scientists such as myself. I would like to honour Gareth by highlighting a few of my research endeavours in which electron microscopy has played a crucial role.

My research team has been exploring how the microstructure and properties of ceramics and metals can be explained by the presence of different grain boundary structures and compositions [1, 2]. We have defined these grain boundary structures as complexions (i.e., the equilibrium state of a grain boundary) [3]. Electron microscopy has been an instrumental tool for this research, and electron micrographs clearly show that grain boundaries undergo phase-like (i.e., complexion) transitions. Some of the first complexions that we observed were "intergranular films" (IGFs) in doped alumina [1], a structure that was originally identified by Thomas and Clarke in $\mathrm{MgO}$-doped $\mathrm{Si}_{3} \mathrm{~N}_{4}$ in the late 1970s [4].

My recent foray into metallurgy can also be attributed to Gareth's microscopy influence. We used aberration-corrected STEM to show that the liquid metal embrittlement of $\mathrm{Ni}$ by $\mathrm{Bi}$ is caused by a complexion transition to a Bi-rich bilayer at the grain boundary [2]. Images revealed that the weak Bi-Bi bonding seriously compromises the cohesive strength of Ni grain boundaries. For instance, Figure 1 shows one intact boundary and two occasions where atomic-scale fractures occurred down the centre of the Bi bilayer. This discovery solved a long-standing mystery and would not have been possible without advanced microscopy. STEM is also a critical tool in our studies of nanocrystalline Ni-W alloys. As seen in Figure 2, we have used images at several length scales to identify impurity phases that are frequently overlooked, to track nanoscale particles that stagnate grain growth, and to identify nanocrystalline complexions such as $\mathrm{W}$-segregation to a $\mathrm{Ni}_{4} \mathrm{~W} / \mathrm{WO}_{\mathrm{x}}$ phase boundary.

In a way, my use of electron microscopy to study grain boundary complexions is the interfacial analogue to Gareth's pioneering studies on dual-phase steels [5]. By carefully controlling the kinetic transformation paths, Gareth created steels with extraordinary combinations of strength and ductility. He also developed time-temperature-transformation TTT diagrams that describe the kinetics of high purity kaolinite [6]. Just this year, we published the first paper on the kinetics of grain boundary complexion transitions and established the first TTT diagrams for grain boundaries [7]. I believe that we are adding to Gareth's vision by mapping the kinetic paths of grain boundary transformations.

Finally, we note an emerging field of study called "anti-thermal" behaviour, a potentially transformative phenomenon in which materials rate processes remain constant or even become slower as temperature 
increases [8]. Once again, advanced electron microscopy will be critical to discover which grain boundaries and materials are "anti-thermal". By understanding the mechanistic cause of this unusual behaviour, it may be possible to engineer next-generation materials for high-temperature applications.

Gareth had a remarkable ability to foresee critical materials science problems and skilfully solve them using the electron microscope. His impact on the field is even greater than his many research successes though, because he trained a generation of materials scientists to similarly recognize important problems and to solve them with electron microscopy. I believe that Gareth would be excited about the advances that have been made by applying his approach to the study of grain boundary complexions.

\section{References:}

[1] SJ Dillon et al., Acta Materialia 55 (2007), p. 6208.

[2] J Luo et al., Science 333 (2011), p. 1730.

[3] PR Cantwell et al., Acta Materialia 62 (2014), p. 1.

[4] DR Clarke, G Thomas, Journal of the American Ceramic Society 60 (1977), p. 491.

[5] NJ Kim, G Thomas, Metallurgical Transactions A 12 (1981), p. 483.

[6] K Liu et al., Journal of the American Ceramic Society 77 (1994), p. 1545.

[7] PR Cantwell et al., Acta Materialia 106 (2016), p. 78.

[8] PR Cantwell et al., Scripta Materialia 103 (2015), p. 1.

[9] The authors acknowledge funding from the ONR-MURI program (grant no. N00014-11-0678).

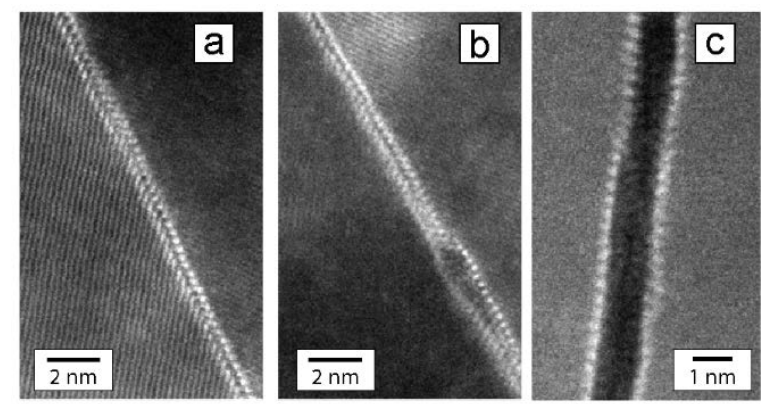

Figure 1. Example of using electron microscopy to solve the problem of liquid metal embrittlement. HAADF images of Bi segregation to grain boundaries in Ni-Bi where the boundary is (a) intact, (b) beginning to rupture, and (c) completely ruptured. Figure adapted from [2].
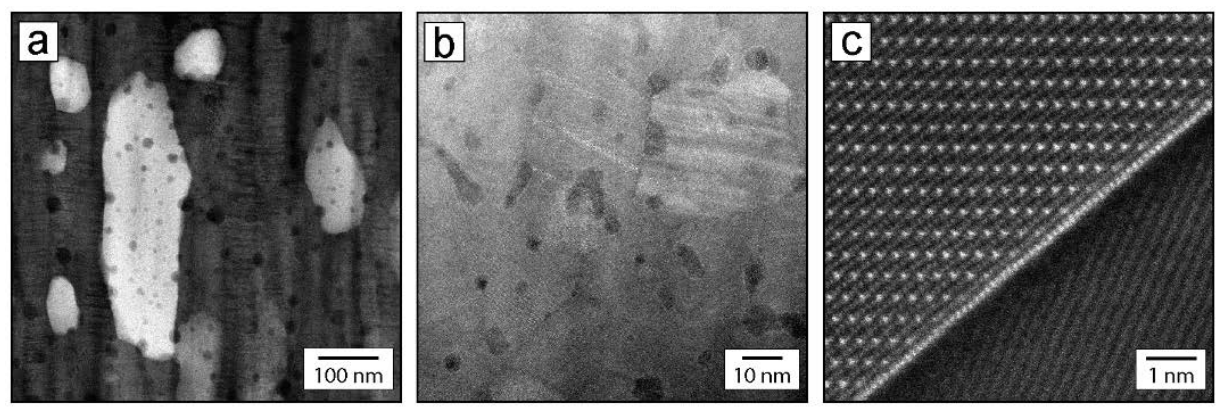

Figure 2. Example of applying electron microscopy to understand the mechanisms of nanocrystalline thermal stability in metals. STEM-HAADF images of nanocrystalline Ni-W alloys that helped identify (a) unexpected impurity $\mathrm{Ni}_{6} \mathrm{~W}_{6} \mathrm{C}$ phases, (b) nanoscale oxides that limited grain growth, and (c) Wsegregation to a $\mathrm{Ni}_{4} \mathrm{~W} / \mathrm{WO}_{\mathrm{x}}$ phase boundary. 